\title{
The Hilbert scheme of space curves sitting on a smooth surface containing a line
}

\author{
Jan O. Kleppe
}

\begin{abstract}
We continue the study of maximal families $W$ of the Hilbert scheme, $\mathrm{H}(d, g)_{s c}$, of smooth connected space curves whose general curve $C$ lies on a smooth degree-s surface $S$ containing a line. For $s \geq 4$, we extend the two ranges where $W$ is a unique irreducible (resp. generically smooth) component of $\mathrm{H}(d, g)_{s c}$. In another range, close to the boarder of the nef cone, we describe for $s=4$ and 5 components $W$ that are non-reduced, leaving open the non-reducedness of only 3 (resp. 2) families for $s \geq 6$ (resp. $s=5$ ), thus making progress to recent results of Kleppe and Ottem in [28]. For $s=3$ we slightly extend previous results on a conjecture of non-reduced components, and in addition we show its existence in a subrange of the conjectured range.
\end{abstract}

AMS Subject Classification. 14C05 (Primary), 14C20, 14K30, 14J28, 14H50 (Secondary). Keywords. Space curves, Quartic surfaces, Cubic surfaces, Hilbert scheme, Hilbert-flag scheme, relative Picard scheme

\section{Introduction}

Let $\mathrm{H}(d, g)_{s c}$ be the Hilbert scheme of smooth connected curves in $\mathbb{P}^{3}$. In this paper we study irreducible, possibly non-reduced, components of $\mathrm{H}(d, g)_{s c}$ whose general curve sits on a smooth surface containing a line. Since the first example of a non-reduced component was found by Mumford [34] there has been many geometers who were challenged by this phenomena and quite a lot of papers has appeared that consider the problem of non-reducedness and related questions, see e.g. [4, 6, 7, 13, 17], [21, 23, 25, 26], [31, 32], [33, 35, 36] and the books [29] and [20].

A classical way of analyzing whether the closure of a family is a component, possibly nonreduced, is to take a general curve $C$ of a family and describe the curves in an open neighborhood of $(C) \in \mathrm{H}(d, g)_{s c}$. More recently several authors has been able to sufficiently describe the obstruction of deforming $C$ and conclude similarly [13,26,32,33,35,36]. In the recent paper [28] we find non-reduced, as well as generically smooth, irreducible components of $\mathrm{H}(d, g)_{s c}$, and we prove non-reducedness along the classical line. This works quite well if the genus is large and the minimal degree $s(C)$ of a surface containing a general curve $C$ is small (as in [17]).

The families we consider are $s$-maximal families. To define them let $W$ is an irreducible closed subset of $\mathrm{H}(d, g)_{s c}$ and let $s(W):=s(C)$ where $C$ is a general curve of $W$. As in [21] we say $W$ is $s(W)$-maximal if it is maximal with respect to $s(W)$, i.e. $s(V)>s(W)$ for any closed irreducible subset $V$ properly containing $W$. If $d>s^{2}$, an $s$-maximal family containing $(C)$ is nothing but the image under the forgetful morphism $p r_{1}: \mathrm{D}(d, g ; s)_{s c} \rightarrow \mathrm{H}(d, g)_{s c},(C, S) \mapsto(C)$ of an irreducible component of the Hilbert-flag scheme $\mathrm{D}(d, g ; s)_{s c}$ containing $(C, S)$, see Section 2 for details.

An $s$-maximal family $W$ needs not be a component of $\mathrm{H}(d, g)_{s c}$. Indeed $4 d \leq \operatorname{dim} W$ is obviously a necessary condition for $W$ to be a component, while $H^{1}\left(\mathcal{I}_{C}(s)\right)=0, \mathcal{I}_{C}$ the sheaf ideal of $C \subset \mathbb{P}^{3}$, turns out to be a sufficient condition because $p r_{1}$ is smooth at $(C, S)$. When $H^{1}\left(\mathcal{I}_{C}(s)\right)=0, W$ is even generically smooth if the corresponding component of $\mathrm{D}(d, g ; s)_{s c}$ is, e.g. if $s \leq 4$. Since the dimension $\operatorname{dim} W$ of an $s$-maximal family is easy to compute for $s \leq 4$, we get in particular that $g \geq g_{1}:=3 d-18$ is necessary (for $d>9$ and $S$ smooth) while $g>g_{2}:=\left\lfloor\left(d^{2}-4\right) / 8\right\rfloor$ is sufficient 
for a 3-maximal family $W$ to be an irreducible component. Indeed $H^{1}\left(\mathcal{I}_{C}(3)\right)=0$ holds for $g>g_{2}$ by [21, Cor. 17]. Thus $W \subset \mathrm{H}(d, g)_{s c}$ may be a non-reduced component only if $g_{1} \leq g \leq g_{2}$. The author conjectured in 22 , that $H^{1}\left(\mathcal{I}_{C}(3)\right) \neq 0, d \geq 14$ and $g_{1} \leq g \leq g_{2}$ imply that a 3-maximal family is a non-reduced irreducible component of $\mathrm{H}(d, g)_{s c}$ and proved it in some subrange. Ellia extended the range substantially in [7, and pointed out that the conjecture is false unless we also suppose $H^{1}\left(\mathcal{I}_{C}(1)\right)=0$. Looking more closely to Ellia's proof in [7], we extend the modified conjecture (assuming also $H^{1}\left(\mathcal{I}_{C}(1)\right)=0$ ) further in Theorem 4.5, and we prove the existence of such components in a range covering all cases where the conjecture is proven (Theorem 4.7).

All components so far (i.e. for $s=3$ ) are irreducible components $W$ of $\mathrm{H}(d, g)_{s c}$ whose general curve sits on a smooth surface containing a line $\Gamma$. Motivated by the theory of the Noether-Lefschetz locus (NL), the components of NL whose general surface $S$ is smooth and contains a line are very special ( [12, 14, 38]). In Section 3 we explicitly describe $s$-maximal families for $s \geq 4$ on such surfaces $S \supset \Gamma$ with Picard number 2. If $d>s^{2}$ and $C \equiv a \Gamma+b(H-\Gamma), H$ a hyperplane section, is a general curve of $W$ and $a>s-4$ (resp. $a>s$ ), then $W$ is a unique irreducible (resp. generically smooth) component of $\mathrm{H}(d, g)_{s c}$ except possibly in two cases. There are natural maps $\alpha_{C}: H^{0}\left(S, \mathcal{N}_{S}\right) \rightarrow H^{1}\left(C, \mathcal{N}_{C / S}\right)$, where $\operatorname{coker}\left(\alpha_{C}\right)$ is the obstruction space of $\mathrm{D}(d, g ; s)_{s c}$ at $(C, S)$, and $\gamma_{C}: H^{0}\left(\mathcal{N}_{C}\right) \rightarrow H^{1}\left(\mathcal{I}_{C}(s)\right)$ that infinitesimally determine $p r_{1}$ at $(C, S)$ (see (2) below). In [28, Thms. 1.1, 1.2, 7.3] we computed $\operatorname{coker}\left(\alpha_{C}\right)$ and hence $\operatorname{dim} V$, and we showed that $W$ is a unique (resp. generically smooth) component under a little too restrictive assumptions, see Theorem 3.4 for the new version and Remark 3.5 for the generalizations in this paper compared to 28 , Thms. 1.1, 1.2, 7.3]. In particular, also for $s \geq 5$ there are only three very explicitly described families (corresponding to $H^{1}\left(\mathcal{I}_{C}(s)\right) \neq 0$ ), that may correspond to non-reduced components, otherwise $W$ is a generically smooth component in $\mathrm{H}(d, g)_{s c}$. Thus we extend in Theorem 3.4 (i) and (ii) [28] in a good number of cases. For the three families we still lack sufficient information of the map $\gamma_{C}$ to state that all these components are non-reduced. We expect that they are non-reduced components which we prove for $s=4$ and for one family for $s=5$, and in general provided $\gamma_{C}$ is non-zero.

While we should have liked to understand the map $\gamma_{C}$ better, the other important map $\alpha_{C}$ is under the assumption $H^{1}\left(\mathcal{I}_{C}(s-4)\right)=0$ given by its partner for the relative Picard scheme, Pic. In fact the rational morphism $\pi: \mathrm{D}(d, g ; s)_{s c}-\rightarrow$ Pic, $(C, S) \mapsto\left(\mathcal{O}_{S}(C), S\right)$ is smooth at $(C, S)$ provided $H^{1}\left(\mathcal{I}_{C}(s-4)\right)=0$ ( [15, Rem. 4.5]). The smoothness of the morphisms $p r_{1}$ and $\pi$, as well as [12, Prop. 1.5]) are important in the proof of Lemma 2.1 and hence of the theorems. In particular the comparison between $\mathrm{H}(d, g)$ and Pic works very well under the assumption $H^{1}\left(\mathcal{I}_{C}(s)\right)=0=$ $H^{1}\left(\mathcal{I}_{C}(s-4)\right)$ which we in Section 2 illustrate by giving an example (Remark 2.2) of a non-reduced component of Pic.

We thank O. A. Laudal for interesting discussions on deformations and Hilbert-flag schemes and J. C. Ottem for working together in [28] which has inspired this paper.

\subsection{Notations and terminology}

In this paper the ground field $k$ is algebraically closed of characteristic zero (and equal to the complex numbers when the concept "very general" is used). A surface $S$ in $\mathbb{P}^{3}$ is a hypersurface, and a curve $C$ in $\mathbb{P}^{3}$ (resp. in $S$ ) is a pure one-dimensional subscheme of $\mathbb{P}:=\mathbb{P}^{3}$ (resp. $S$ ) with ideal sheaf $\mathcal{I}_{C}$ (resp. $\mathcal{I}_{C / S}$ ) and normal sheaf $\mathcal{N}_{C}=\mathcal{H o m}$ OP $_{\mathbb{P}}\left(\mathcal{I}_{C}, \mathcal{O}_{C}\right)\left(\right.$ resp. $\mathcal{N}_{C / S}=\operatorname{Hom}_{\mathcal{O}_{S}}\left(\mathcal{I}_{C / S}, \mathcal{O}_{C}\right)$ ). If $\mathcal{F}$ is a coherent $\mathcal{O}_{\mathbb{P}}$-Module, we let $H^{i}(\mathcal{F})=H^{i}(\mathbb{P}, \mathcal{F}), h^{i}(\mathcal{F})=\operatorname{dim} H^{i}(\mathcal{F}), \chi(\mathcal{F})=\Sigma(-1)^{i} h^{i}(\mathcal{F})$ and $s(C)=\min \left\{n \mid h^{0}\left(\mathcal{I}_{C}(n)\right) \neq 0\right\}$. We denote by $\mathrm{H}(d, g)$ (resp. $\mathrm{H}(d, g)_{s c}$ ) the Hilbert scheme of (resp. smooth connected) space curves of Hilbert polynomial $\chi\left(\mathcal{O}_{C}(t)\right)=d(C) t+1-g(C)$, and we let $d:=d(C)$ and $g:=g(C)$. A curve $C$ is called unobstructed if $\mathrm{H}(d, g)$ is smooth at the corresponding point $(C)$. A curve in a small enough open irreducible subset $U$ of $\mathrm{H}(d, g)$ is called a general curve of 
$\mathrm{H}(d, g)$. A generization $C^{\prime} \subset \mathbb{P}^{3}$ of $C \subset \mathbb{P}^{3}$ in $\mathrm{H}(d, g)$ is the general curve of some irreducible subset of $\mathrm{H}(d, g)$ containing $(C)$. By an irreducible component of $\mathrm{H}(d, g)$ we always mean a non-embedded irreducible component. A member of a closed irreducible subset $V$ of $\mathrm{H}(s)$ or $\mathrm{H}(d, g)_{s c}$ is called very general in $V$ if it is smooth and sits outside a countable union of proper closed subset of $V$.

\section{Background}

In the following we recall some results from the the background section of [28] that extend ideas and results appearing in [21], 25] and [24] and that use the deformation theory developed by Laudal in [29]; in particular the results rely on [29, Thm. 4.1.14]. Moreover ideas in [15] and [12] are central.

\subsection{The Hilbert flag scheme and the relative Picard scheme}

Let $\mathrm{D}(d, g ; s)$ be the Hilbert-flag scheme parameterizing pairs $(C, S)$ of curves $C$ contained in a degree $s$-surface $S \subset \mathbb{P}^{3}$ where $d$ and $g$ are the degree and genus of $C$. If $S$ is smooth then $\mathcal{N}_{C / S} \simeq \omega_{C} \otimes \omega_{S}^{-1}$ and we have a connecting homomorphism $\delta: H^{0}\left(\left.\mathcal{N}_{S}\right|_{C}\right) \rightarrow H^{1}\left(\mathcal{N}_{C / S}\right) \simeq H^{0}\left(\mathcal{O}_{C}(s-4)\right)^{\vee}$ induced by the sequence $\left.0 \rightarrow \mathcal{N}_{C / S} \rightarrow \mathcal{N}_{C} \rightarrow \mathcal{N}_{S}\right|_{C} \rightarrow 0$ of normal bundles. Let $\alpha_{C}:=\delta \circ m$ be the composed map of the natural restriction $m: H^{0}\left(\mathcal{N}_{S}\right) \rightarrow H^{0}\left(\left.\mathcal{N}_{S}\right|_{C}\right)$ with $\delta$, let $A^{2}:=$ coker $\alpha$ and let $A^{1}$ be the tangent space of $\mathrm{D}(d, g ; s)$ at $(C, S)$. Then the tangent map $A^{1} \rightarrow H^{0}\left(\mathcal{N}_{C}\right)$ of the $1^{\text {st }}$ projection,

$$
p r_{1}: \mathrm{D}(d, g ; s) \longrightarrow \mathrm{H}(d, g), \quad \text { induced by } \quad \operatorname{pr}_{1}\left(\left(C_{1}, S_{1}\right)\right)=\left(C_{1}\right) \text {, }
$$

at $(C, S)$ fits into an exact sequence

$$
0 \rightarrow H^{0}\left(\mathcal{I}_{C / S}(s)\right) \rightarrow A^{1} \rightarrow H^{0}\left(\mathcal{N}_{C}\right) \rightarrow H^{1}\left(\mathcal{I}_{C}(s)\right) \rightarrow \operatorname{coker} \alpha_{C} \rightarrow H^{1}\left(\mathcal{N}_{C}\right) \rightarrow H^{1}\left(\mathcal{O}_{C}(s)\right) \rightarrow 0
$$

from which we deduce $\operatorname{dim} A^{1}-\operatorname{dim} A^{2}=(4-s) d+g+\left(\begin{array}{c}s+3 \\ 3\end{array}\right)-2$. Note that $p r_{1}$ is a projective morphism ( [20, Thm. 24.7]). By [21, Lem. A10] $p r_{1}$ is smooth at $(C, S)$ if $H^{1}\left(\mathcal{I}_{C}(s)\right)=0$. Moreover $A^{2}=$ coker $\alpha_{C}$ contains the obstructions of deforming the pair $\left.(C, S)([21,(2.6)])\right)$, and we have $A^{2}=0$ for $s \leq 4$ if $C$ is smooth and connected and not a complete intersection (c.i.) in $S$ (by the infinitesimal Noether-Lefschetz theorem if $s=4$ and because $\delta=0$ if $s \leq 3$ ). Let $d>s^{2}$. Restricting $p r_{1}$ to the open set $\mathrm{D}(d, g ; s)_{s c}$ where the curves are smooth and connected, we get that an $s$-maximal family $W$ of $\mathrm{H}(d, g)_{s c}$ containing $(C)$ is nothing but the image under $p r_{1}$ of an irreducible component of $\mathrm{D}(d, g ; s)_{s c}$ containing $(C, S)$ ( [23, Def. 1.24 and Cor. 1.26]).

We also need to consider the Hilbert scheme, $\mathrm{H}(s) \simeq \mathbb{P}^{\left(\begin{array}{c}s+3 \\ 3\end{array}\right)-1}$, of degree- $s$ surfaces in $\mathbb{P}^{3}$, the $2^{\text {nd }}$ projection $p r_{2}: \mathrm{D}(d, g ; s) \longrightarrow \mathrm{H}(s)$, induced by $p r_{2}\left(\left(C_{1}, S_{1}\right)\right)=\left(S_{1}\right)$, and the relative Picard scheme, Pic, over the open set in $\mathrm{H}(s)$ of smooth surfaces of degree $s$. There is a projection $p_{2}$ : $\mathrm{Pic} \rightarrow \mathrm{H}(s)$, forgetting the invertible sheaf, and a rational map:

$$
\pi: \mathrm{D}(d, g ; s)--\rightarrow \text { Pic }, \quad \text { induced by } \pi\left(\left(C_{1}, S_{1}\right)\right)=\left(\mathcal{O}_{S_{1}}\left(C_{1}\right), S_{1}\right)
$$

defined over the open subset $U \subset \mathrm{D}(d, g ; s)$ given by pairs $\left(C_{1}, S_{1}\right)$ where $C_{1}$ is Cartier on a smooth $S_{1}$. Obviously, if we restrict to $U$ we have $p_{2} \circ \pi=p r_{2}$. If $H^{1}\left(S, \mathcal{O}_{S}(C)\right)=0$ then $\pi$ is smooth at $(C, S)$ by [15, Rem. 4.5]. Indeed, $H^{1}(S, \mathcal{L})=0, \mathcal{L}:=\mathcal{O}_{S}(C)$, implies a surjective map $A^{1} \rightarrow T_{\text {Pic }, \mathcal{L}}$ between the tangent spaces of $\mathrm{D}(d, g ; s)$ at $(C, S)$ and Pic at $(\mathcal{L})$ and an injection coker $\alpha_{C} \rightarrow$ coker $\alpha_{\mathcal{L}}$ of their obstruction spaces where $\alpha_{\mathcal{L}}$ is the composition of $\alpha_{C}$ with the connecting homomorphism $H^{1}\left(\mathcal{N}_{C / S}\right) \rightarrow H^{2}\left(\mathcal{O}_{S}\right)$ induced from the exact sequence $0 \rightarrow \mathcal{O}_{S} \rightarrow \mathcal{O}_{S}(C) \rightarrow \mathcal{N}_{C / S} \rightarrow 0$ (cf. [12, Thm. 1], 25, Sect. 4] and [24]). Noticing that $H^{1}\left(S, \mathcal{O}_{S}(C)\right) \simeq H^{1}\left(\mathbb{P}^{3}, \mathcal{I}_{C}(s-4)\right)^{\vee}$, we have 
Lemma 2.1. Let $S \subset \mathbb{P}^{3}$ be a smooth degree-s surface, $H$ a hyperplane section, let $E$ and $C$ be curves on $S$ satisfying $C \equiv$ (i.e. linearly equivalent to) $e E+f H$ for some $e \neq 0, f \in \mathbb{Z}$.

(i) Suppose $H^{1}\left(\mathcal{I}_{E}(s-4)\right)=H^{1}\left(\mathcal{I}_{C}(s-4)\right)=0$. Then $\mathrm{D}(d, g ; s)$ is smooth at $(C, S)$ if and only if $\mathrm{D}(d(E), g(E) ; s)$ is smooth at $(E, S)$, and we have

$$
\operatorname{dim} \operatorname{coker} \alpha_{C}+h^{0}\left(\mathcal{I}_{C / S}(s-4)\right)=\operatorname{dim} \operatorname{coker} \alpha_{E}+h^{0}\left(\mathcal{I}_{E / S}(s-4)\right)
$$

(ii) If $H^{1}\left(\mathcal{I}_{E}(s)\right)=0=H^{1}\left(\mathcal{I}_{E}(s-4)\right)=H^{1}\left(\mathcal{I}_{C}(s-4)\right)$ and $\mathrm{H}(d(E), g(E))_{s c}$ is a smooth irreducible scheme and $(E) \in \mathrm{H}(d(E), g(E))_{s c}$, then $\mathrm{D}(d, g ; s)$ is smooth at $(C, S)$ and every $\left(C^{\prime}, S^{\prime}\right) \in \mathrm{D}(d, g ; s)$ satisfying $C^{\prime} \equiv e E^{\prime}+f H^{\prime}$ for some $\left(E^{\prime}, S^{\prime}\right) \in \mathrm{D}(d(E), g(E) ; s)_{s c}, H^{\prime}$ a hyperplane section of a smooth surface $S^{\prime} \subset \mathbb{P}^{3}$, belongs to the unique irreducible component of $\mathrm{D}(d, g ; s)$ containing $(C, S)$.

Proof. The main points in the proof is that $\pi$ is smooth at $(C, S)$ and that $\alpha_{\mathcal{O}_{S}(C)}=e \cdot \alpha_{\mathcal{O}_{S}(E)}$ ( [12, Prop. 1.5 and Constr. 1.8]) leads to an isomorphism of their cokernels, see [28, Lem. 2.2].

Remark 2.2 (Example of a non-reduced irreducible component of the relative Picard scheme Pic).

If both $H^{1}\left(\mathcal{I}_{C}(s)\right)$ and $H^{1}\left(\mathcal{I}_{C}(s-4)\right)$ vanish, then the morphisms pr $r_{1}$ of (11) and $\pi$ of (3) are smooth at $(C, S)$. Using this we get that many properties of the Hilbert scheme $\mathrm{H}(d, g)_{s c}$ at $(C)$ are transferred to the relative Picard scheme Pic at $\left(\mathcal{O}_{S}(C), S\right)$, and vice versa. For instance if we take the general curve $C$ of the non-reduced component $V \subset \mathrm{H}(14,24)_{s c}$ of Mumford, and a smooth general surface $S$ of degree 6 containing $C$, then $\left(\mathcal{O}_{S}(C), S\right)$ is the general point of a non-reduced irreducible component $P$ of Pic. This follows from the fact that smooth morphisms take generic (resp. smooth) points onto generic (resp. smooth) points. Of course we have to verify the assumptions;

$$
H^{1}\left(\mathcal{I}_{C}(s)\right)=H^{1}\left(\mathcal{I}_{C}(s-4)\right)=0 \quad \text { for } \quad s=6
$$

with $S$ smooth and $(C, S)$ a general point of $\mathrm{D}(d, g ; s)$. For the general curve in Mumford's example it is known that the homogeneous ideal $I(C)$ allows 4 minimal generators of degree $\{3,6,6,6\}$ that correspond to smooth surfaces by [2] and that $H^{1}\left(\mathcal{I}_{C}(v)\right)=0$ for $v \notin\{3,4,5\}$. Hence we may take the general degree-6 surface containing $C$ to be smooth. Thus we conclude that $P$ is a non-reduced irreducible component of Pic, see [1, 3, 4] for a comparison with the Noether-Lefschetz locus.

We will also need the following lemma (cf. [30, Cor. II 3.8] and see [30, Thm. II 3.1] for a proof).

Lemma 2.3. (A. Lopez) Let $E \subset \mathbb{P}^{3}$ be a smooth irreducible curve, let $n \geq 4$ be an integer and suppose the degree of every minimal generator of the homogeneous ideal of $E$ is at most $n-1$. Let $S$ be a very general smooth surface of degree $n$ containing $E$ and let $H$ be a hyperplane section. Then $\operatorname{Pic}(S) \simeq \mathbb{Z} \oplus \mathbb{Z}$ and we may take $\left\{\mathcal{O}_{S}(H), \mathcal{O}_{S}(E)\right\}$ as a $\mathbb{Z}$-basis for $\operatorname{Pic}(S)$.

\subsection{On the maximum genus of space curves}

Finally let us recall the definition of the maximum genus, $G(d, s)$, of smooth connected space curves of degree $d$ not contained in a surface of degree $s-1$, cf. [16]. By definition,

$$
G(d, s)=\max \left\{g(C) \mid(C) \in \mathrm{H}(d, g)_{s c} \text { and } H^{0}\left(\mathcal{I}_{C}(s-1)\right)=0\right\} .
$$

In the C-range, i.e. when $d>s(s-1)$, Gruson and Peskine showed in [16] that

$$
G(d, s)=1+\frac{d}{2}\left(\frac{d}{s}+s-4\right)-\frac{r(s-r)(s-1)}{2 s} \quad \text { where } d+r \equiv 0 \bmod s \text { for } 0 \leq r<s
$$


and that $g(C)=G(d, s)$ if and only if $C$ is directly linked to a plane curve $E$ of degree $r$ by a c.i. of type $(s, f), f:=(d+r) / s$. In the "extended $C$-range": $s(s-1) \geq d \geq s^{2}-2 s+2$ where the upper part of the $B$-range is included, letting $\mu:=d-\left(s^{2}-2 s+3\right)$, one knows that ( [18, Thm. A])

$$
\begin{gathered}
G(d, s)=s^{3}-5 s^{2}+9 s-6+\frac{\mu(\mu+2 s-3)}{2} \text { for } \quad s-3 \geq \mu \geq 0, \text { and } \\
G(d, s)=1+d(s-3) \quad \text { for } \quad d=s^{2}-2 s+2, \text { i.e. for } \quad \mu=-1 .
\end{gathered}
$$

Moreover, if $g(C)=G(d, s)$, then $C$ is ACM with $s(C)=s$ in (6), and $C$ is a zero-section of the null correlation bundle twisted by $s-1$ in (77).

\section{Components of $\mathrm{H}(d, g)_{s c}$ for $s \geq 4$, the surface contains a line}

Let $S$ be a smooth surface of degree $s \geq 4$ in $\mathbb{P}^{3}$ defined by a form $x_{0} P+x_{1} Q$, where $P, Q$ are very general homogeneous polynomials of degree $s-1$. Families of curves on such surfaces were studied in Theorems 1.1, 1.2 and 7.3 of [28]. Here we improve upon these results.

Let $\Gamma_{1}=\left\{x_{0}=x_{1}=0\right\}$ and $\Gamma_{2}=\left\{x_{0}=Q=0\right\}$. The hyperplane section satisfies $H \equiv \Gamma_{1}+\Gamma_{2}$, $H^{2}=s$ and we may suppose $\operatorname{Pic}(S) \simeq \mathbb{Z} \Gamma_{1} \oplus \mathbb{Z} H$ by Lemma 2.3 and that $\Gamma_{2}$ is a smooth connected curve. If $C \equiv a \Gamma_{1}+b \Gamma_{2}$ then $d=C \cdot H, K=(s-4) H$ and the adjunction formula which implies that the intersection matrix is $\left(\Gamma_{i} \cdot \Gamma_{j}\right)=\left(\begin{array}{cc}2-s & s-1 \\ s-1 & 0\end{array}\right)$, leads also to

$$
d=a+(s-1) b \text { and } g=1+(s-1) a b+\frac{1}{2}\left((s-4) a+(s-4)(s-1) b-(s-2) a^{2}\right) .
$$

Lemma 3.1. Let $S$ be a smooth surface of degree $s$ with $\Gamma_{1}, \Gamma_{2}$ as above. It holds:

(i) Any effective divisor on $S$ is linearly equivalent to $a \Gamma_{1}+b \Gamma_{2}$ where $a, b \geq 0$.

(ii) Every nef divisor is linearly equivalent to $a \Gamma_{1}+b \Gamma_{2}$ where $(s-1) b \geq(s-2) a \geq 0$.

(iii) The divisor $D=(s-1) \Gamma_{1}+(s-2) \Gamma_{2}$ is base-point free.

(iv) Any divisor $C$ satisfying $C \cdot \Gamma_{1} \geq 0$ and $C \cdot \Gamma_{2} \geq 0$ is nef and base-point free.

(v) If a divisor $C$ satisfies $C \cdot \Gamma_{1} \geq 0$ and $C \cdot \Gamma_{2}>0$ then $|C|$ contains a smooth irreducible curve.

See [28] for a proof. Now the Kawamata-Viehweg vanishing theorem, for $D \equiv a \Gamma_{1}+b \Gamma_{2}$, implies:

$$
H^{i}\left(S, \mathcal{O}_{S}(D)\right)=0 \text { for } i>0 \text { provided } a>s-4 \text { and }(s-1) b \geq(s-2) a+s-4
$$

because the assumptions on $a, b$ imply that $D-K$ is nef and big. From this, we got Theorem 7.3 of [28]. Here we generalize (9) leading to a significant improvement of that result, cf. [28, Lem. 2.5]. Indeed we have

Lemma 3.2. Let $S$ be a smooth surface of degree $s$ with $\Gamma_{1}, \Gamma_{2}$ as above and let $C$ be a divisor linearly equivalent to $a \Gamma_{1}+b \Gamma_{2}$ where $(s-1) b-(s-2) a=t$ with $t \geq-2$. Moreover suppose $a>s-4$. Then

(i) $H^{i}\left(S, \mathcal{O}_{S}(C)\right)=0$ for $i>0$ provided $t>-2$ or $(t=-2$ and $a=s-3)$, and

(ii) $H^{1}\left(S, \mathcal{O}_{S}(C)\right) \simeq k, H^{2}\left(S, \mathcal{O}_{S}(C)\right)=0$ provided $t=-2$ and $a \neq s-3$. 
Proof. Firstly we suppose $a>s-3$. To apply (9) onto $D=C-\Gamma_{1}$, we notice that

$$
(s-1) b=(s-2) a-(s-2)+s-2+t \geq(s-2)(a-1)+s-4
$$

by assumption. Thus $H^{i}\left(S, \mathcal{O}_{S}\left(C-\Gamma_{1}\right)\right)=0$ for $i>0$ by (9). Moreover since

$$
0 \rightarrow \mathcal{O}_{S}\left(C-\Gamma_{1}\right) \rightarrow \mathcal{O}_{S}(C) \rightarrow \mathcal{O}_{\Gamma_{1}}\left(C \cdot \Gamma_{1}\right) \rightarrow 0
$$

is exact, we deduce the exact sequence

$$
\rightarrow H^{1}\left(\mathcal{O}_{S}\left(C-\Gamma_{1}\right)\right) \rightarrow H^{1}\left(\mathcal{O}_{S}(C)\right) \rightarrow H^{1}\left(\mathcal{O}_{\mathbb{P}^{1}}\left(C \cdot \Gamma_{1}\right)\right) \rightarrow H^{2}\left(\mathcal{O}_{S}\left(C-\Gamma_{1}\right)\right) \rightarrow H^{2}\left(\mathcal{O}_{S}(C)\right) \rightarrow 0
$$

where $C \cdot \Gamma_{1}=-a(s-2)+b(s-1)=t$ and we easily get the lemma in the case $a \neq s-3$.

Finally if $a=s-3$ we apply (9) onto $D:=C$. Since we have $a>s-4$ it suffices to show $(s-1) b \geq(s-2)(s-3)+s-4$, or equivalently $b \geq s-3-1 /(s-1)$ which holds if $b \geq s-3$. Since $b \geq s-4$ follows from the assumption $(s-1) b=(s-2) a+t \geq(s-2)(s-3)+(-2)$ and the curve $\Gamma_{1}+(s-4) H \equiv(s-3, s-4)$ is ACM, we get the lemma.

The assumption $t \geq-2$ in Lemma 3.2 may be weakened. Indeed we have

Lemma 3.3. Let $S$ be a smooth surface of degree $s$ with $\Gamma_{1}, \Gamma_{2}$ as above, let $C \equiv a \Gamma_{1}+b \Gamma_{2}$ and $t:=(s-1) b-(s-2) a$ and suppose $-1 \geq t \geq-4, a>s-2, s \geq 4$ and $(t, s) \neq(-4,4)$ (resp. $(t, s)=(-4,4))$. Then

$$
\operatorname{dim} H^{1}\left(S, \mathcal{O}_{S}(C)\right)=-t-1(\operatorname{resp} .4 \text { if }(t, s)=(-4,4)) .
$$

Proof. Suppose $(t, s) \neq(-4,4)$. Then we have $H^{i}\left(S, \mathcal{O}_{S}\left(C-\Gamma_{1}\right)\right)=0$ for $i>0$ by Lemma 3.2 since $a-1>s-3$ and $(s-1) b-(s-2)(a-1)=t+s-2>-2$, i.e. the assumptions of the lemma are fulfilled for $(a-1) \Gamma_{1}+b \Gamma_{2}$. By (10), we get $h^{1}\left(\mathcal{O}_{S}(C)\right)=h^{1}\left(\mathcal{O}_{\mathbb{P}^{1}}\left(C \cdot \Gamma_{1}\right)\right)=-t-1$ because $C \cdot \Gamma_{1}=t$. If $(t, s)=(-4,4)$, then Lemma 3.2 yields $H^{1}\left(S, \mathcal{O}_{S}\left(C-\Gamma_{1}\right)\right) \simeq k$ and we conclude by (10).

We are now ready to generalize [28, Thms. 1.1, 1.2, 7.3]. Below we often write $(a, b)$ for $a \Gamma_{1}+b \Gamma_{2}$.

Theorem 3.4. Let $S \subset \mathbb{P}^{3}$ be a smooth degree-s surface containing a line $\Gamma_{1}$, let $\Gamma_{2} \equiv H-\Gamma_{1}$ be a smooth curve and suppose $\operatorname{Pic}(S) \simeq \mathbb{Z} \Gamma_{1} \oplus \mathbb{Z} \Gamma_{2}$ (e.g. $S$ is very general) and $s \geq 4$. Let $C \equiv a \Gamma_{1}+b \Gamma_{2}$ be a smooth connected curve of degree $d>s^{2}$ with $a \neq b$.

(i) Suppose $a>s-4$. Then $C$ belongs to a unique $s$-maximal family $W \subset \mathrm{H}(d, g)_{s c}$. Moreover if $\tilde{S}$ is a degree-s surface containing a very general member of $W$, then $\operatorname{Pic}(\tilde{S})$ is freely generated by the classes of a line and a smooth plane degree- $(s-1)$ curve, and every $C \equiv a \Gamma_{1}+b \Gamma_{2}$ contained in some surface $S$ as above belongs to $W$. Furthermore

$$
\operatorname{dim} W=(4-s) d+g+\left(\begin{array}{c}
s+3 \\
3
\end{array}\right)+\left(\begin{array}{c}
s-1 \\
3
\end{array}\right)-s+1 \text { with d,g as in (8), }
$$

and if $(s, a, b) \notin\{(4,6,4),(4,9,6)\}$ then $W$ is an irreducible component of $\mathrm{H}(d, g)_{s c}$.

(ii) Suppose $s<a<\frac{(s-1) b-2}{s-2}$ or $(a, b)=(s+1, s)$. Then $W$ is moreover a generically smooth irreducible component of $\mathrm{H}(d, g)_{s c}$. 
(iii) Suppose $\frac{(s-1) b-2}{s-2} \leq a \leq \frac{(s-1) b}{s-2},(a, b) \neq(s+1, s)$ and $(s, a, b) \notin\{(4,6,4),(4,9,6)\}$. Then $W$ is a non-reduced irreducible component of $\mathrm{H}(d, g)_{\text {sc }}$ provided $h^{0}\left(\mathcal{N}_{C}\right)>\operatorname{dim} W$, or equivalently, provided the map $H^{0}\left(\mathcal{N}_{C}\right) \rightarrow H^{1}\left(\mathcal{I}_{C}(s)\right)$ appearing in (2) is non-zero for the general curve $C$ of $W$. In particular $W$ is a non-reduced irreducible component of $\mathrm{H}(d, g)_{s c}$ if $s=4$, or $s=5$ and $(a, b)=(4 k, 3 k), k \geq 2$.

Remark 3.5. (A) Note that for any $C \equiv(a, b)$ of the theorem we have $a \leq \frac{(s-1) b}{s-2}$ because $C$ is nef. (B) Since the assumption in (iii) and $d>s^{2}$ imply $a>s$, there are exactly three families,

$$
(a, b)=((s-1) n-\mu,(s-2) n-\mu+1), \quad n \geq 3
$$

corresponding to (a): $\mu=s-3$, (b): $\mu=s-2$ and (c): $\mu=s-1$ respectively, that are covered by (iii) of Theorem 3.4 (where (c) is on the border of the nef cone). Thanks to Lemma 3.3 we have

$$
h^{1}\left(\mathcal{I}_{C}(s)\right)=1\left(\text { resp. } h^{1}\left(\mathcal{I}_{C}(s)\right)=2, h^{1}\left(\mathcal{I}_{C}(s)\right)=3\right) \text { for the family (a) (resp. (b), (c)) }
$$

if $s \geq 5$ for family (c). We expect that the corresponding irreducible components of $\mathrm{H}(d, g)_{s c}$ are non-reduced. Indeed they are non-reduced for the family (c) when $s=5$ by Theorem 3.4.

(C) If $s=4$ then Theorem 3.4 implies that the three families described in (B) form non-reduced components (with $n \geq 5$ in (c)). Thus we slightly generalize [28, Thm.1.2 (II)] by including two more cases of non-reduced components. Note that for family $(\mathrm{c}), h^{1}\left(\mathcal{I}_{C}(s)\right)=4$ in this case.

(D) For $s=5$, Theorem 3.4 (ii), resp. (iii) slightly generalize (I), resp. (II) of [28, Thm. 1.2] by including one more infinite family (resp. one more case of a non-reduced component).

(E) For $s \geq 6$ the generalization in Theorem 3.4 of [28, Thm. 7.3] is more substantial because Theorem 3.4 (i), resp. (ii) includes, as s increases, an increasing number of infinite families for which Theorem 3.4 holds (compared with what holds by [28, Thm. 7.3]).

Proof. The assumptions on $a, b$ in (i), resp. (ii), imply that $H^{1}\left(\mathcal{O}_{S}(C)\right)=0$, resp. $H^{1}\left(\mathcal{I}_{C}(s)\right)^{\vee} \simeq$ $H^{1}\left(\mathcal{O}_{S}(C-4 H)\right)=0$ by Lemma 3.2. We therefore get the stated properties of $W$ in (i), resp. (ii) by the same proof is that in [28, Thm. 7.3] (except for $W$ being a component in (i)); the whole point is only that Lemma 3.2 imply the vanishing of $H^{1}\left(\mathcal{O}_{S}(C+v H)\right)$ under weaker assumptions than those used in [28, Thm. 7.3], which lead to corresponding improvements of Theorem 3.4 (i) and (ii).

In (i) it only remains to see that $W$ is an irreducible component. Observe that $H^{1}\left(\mathcal{I}_{C}(s)\right)=0$ does not only prove the generic smoothness of $W$, but it also implies that $W$ is an irreducible component of $H(d, g)_{s c}$. There are, however, cases (i.e. the 3 families mentioned in (B) above) not covered by (ii) of the theorem, and for these we prove that $W$ is an irreducible component as in [28, Thm. 7.3] by e.g. showing $g \geq G(d, s+1)$, except when $(s, a, b) \in\{(4,7,5),(4,12,8),(5,8,6),(6,10,8)\}$. In these four cases we were not able to show that $W$ was an irreducible component in [28, Thms.1.1, 7.3] because $g<G(d, s+1)$. To get (i) and (ii) as stated above it remains to consider them now.

In these cases we suppose there is an irreducible component $V$ of $\mathrm{H}(d, g)_{s c}$ satisfying $W \subset V$ and $\operatorname{dim} W<\operatorname{dim} V$. Since $W$ is $s$-maximal, we may suppose that the general curve $X$ of $V$ satisfies $s(X)>s$.

The case $(a, b)=(12,8)$ and $s=4$. For this class we compute the following numbers: $(d, g)=$ $(36,145), G(d, 5)=147$ and $G(d, 6)=145$, cf. (4). We first consider the option $s(X)=5$. To see that $X$, whence $V$ does not exist we use the theory on Halphen's gaps given in Ellia's paper [8]. If such a curve exists, we compute $r$ in $d(X)+r \equiv 0 \bmod 5$, and we get $r=4=s(X)-1$. Using [8, Prop. IV.3] it follows that there are no such $X$ with maximal numerical character, and by [8, Lem. VI.2] that the genus of $X$ is equal to the genus of the numerical character of $X$. This implies that $X$ is arithmetically Cohen-Macaulay (ACM). 
By Riemann-Roch $\chi\left(\mathcal{I}_{X}(5)\right)=20$, whence $h^{1}\left(\mathcal{O}_{X}(5)\right)=19$ and since $\chi\left(\mathcal{I}_{X}(8)\right)=21$, we get $h^{1}\left(\mathcal{O}_{X}(8)\right) \leq 1$. It follows that $\operatorname{dim}{ }_{0} \operatorname{Hom}_{R}\left(I(X), H_{*}^{1}\left(\mathcal{O}_{X}\right)\right)=19$. Using

$$
h^{0}\left(\mathcal{N}_{X}\right)=4 d+\operatorname{dim}(-4) \operatorname{Hom}_{R}\left(I(X), H_{*}^{1}\left(\mathcal{I}_{X}\right)\right)+\operatorname{dim}{ }_{0} \operatorname{Hom}_{R}\left(I(X), H_{*}^{1}\left(\mathcal{O}_{X}\right)\right)
$$

which holds for curves of maximal rank (cf. Remark 3.6), in particular for ACM curves (where we have $\left.\operatorname{Hom}_{R}\left(I(X), H_{*}^{1}\left(\mathcal{I}_{X}\right)\right)=0\right)$, we conclude that $h^{0}\left(\mathcal{N}_{X}\right)=163<g+33=178$ which contradicts the assumption $\operatorname{dim} W<\operatorname{dim} V$.

Also the case $s(X) \geq 6$ must be considered. Since $g=G(d, 6)=145$ and $d(X) \equiv 0 \bmod 6, X$ is a c.i. of type $(6,6)$ by (5). Since $\chi\left(\mathcal{I}_{X}(6)\right)=12$ we get $h^{1}\left(\mathcal{O}_{X}(6)\right)=10$ and $\operatorname{dim}_{(X)} \mathrm{H}(d, g)=$ $4 d+2 h^{1}\left(\mathcal{O}_{X}(6)\right)=164$, a contradiction. This shows that $W$ is a component.

The case $(a, b)=(7,5)$ and $s=4$. We compute the following numbers: $(d, g)=(22,57)$, $\chi\left(\mathcal{I}_{C}(5)\right)=2, \chi\left(\mathcal{I}_{C}(6)\right)=8$ and $G(d, 5)=58$. Note that $g=G(d, 5)-1$; whence we suppose a generization $X$ of $C$ satisfying $s(X)>4$ exists. Since $H^{1}\left(\mathcal{O}_{C}(6)\right)=0$ by the speciality theorem, (cf. [16]), we get $H^{1}\left(\mathcal{O}_{X}(6)\right)=0$ by semicontinuity and in particular $h^{0}\left(\mathcal{I}_{X}(6)\right) \geq 8$ and $s(X) \leq 6$.

Firstly suppose $s(X)=6$. In this case $X$ belongs to the so-called $B$-range in the classification of curves of maximal genus. Since $\chi\left(\mathcal{I}_{X}(5)\right)=2$, we get $h^{1}\left(\mathcal{O}_{X}(5)\right) \geq 2$. Then [19, Prop. 3.2] implies that $d \geq A(6,5)=23$, a contradiction (see [8] and [19] for the definition of $A(k, f)$ and a discussion on the maximum genus when $d \leq s(s-1)$ ). Or one may use that the maximum genus $G(d, s)$ in range $B$ is known in our case (cf. [19], Cor. 3.8 and Rem. 3.8.1); it is $G(22,6)=55$, as conjectured in [19, Conj. 3.5], whence such an $X$ does not exist.

Secondly suppose $s(X)=5$. Since $d(X)+r \equiv 0 \bmod s(X)$ allows $r=3=s(X)-2$, it follows from [8, Prop. IV.4] that $X$ is bilinked via c.i.'s of type $(5,8)$ and $(5,4)$ to a degree-2 curve $Z$ satisfying $g(Z)=-1$ provided the numerical character is maximal. By [8, Lem. V.1] the minimal resolution of $I(Z)$ is known (the resolution of two skew lines have the same Betti numbers) and the mapping cone construction used twice yields:

$$
0 \rightarrow R(-8) \rightarrow R(-9) \oplus R(-8) \oplus R(-7)^{4} \rightarrow R(-8) \oplus R(-6)^{4} \oplus R(-5) \rightarrow I(X) \rightarrow 0
$$

Thus $X$ is of maximal rank and applying (11), we get $h^{0}\left(\mathcal{N}_{X}\right) \leq 4 d+2=90$ because $\operatorname{dim} H_{*}^{1}\left(\mathcal{I}_{X}\right)=$ $h^{1}\left(\mathcal{I}_{X}(4)\right)=1$ and $h^{1}\left(\mathcal{O}_{X}(5)\right)=1$. Since $\operatorname{dim} W=g+33=90$, we get a contradiction.

Thirdly if the numerical character is not maximal we can again use [8, Lem. VI.2] (or [5]) to see that the genus of $X$ is equal to the genus of the numerical character of $X$. This implies that $X$ is ACM and using (11), we get $h^{0}\left(\mathcal{N}_{X}\right) \leq 4 d+1=89$, i.e. a contradiction.

The case $(a, b)=(8,6)$ and $s=5$. We compute the following numbers: $(d, g)=(32,113)$, and $G(d, 6)=115$. Note that $g=G(d, 6)-2$. Firstly suppose $s(X) \geq 7$. Then $X$ belongs to the $B$-range in the classification of curves of maximal genus. The conjecture in [19, Conj. 3.5] is true for $s(X) \leq 9$ by [19, Rem. 3.8.1]. To find the conjectured value of $G(d, 7)$ we compute $A(7, f)$ for several $f$ to find the largest $f$ satisfying $A(7, f) \leq d$ (see [8] or [19, p. 530] for the definition of $A(s, f)$ and $B(s, f)$ ). We find $A(7,7)=33, A(7,6)=28$ and $B(7,6)=31$, whence $G(d, 7)=111$ by [19, Conj. 3.5] and such a generization $X$ of $C$ does not exists because $g=113$.

Therefore we suppose $s(X)=6$. Since $d(X)+r \equiv 0 \bmod s(X)$ allows $r=4=s(X)-2$, it follows from Ellia's paper [8, Prop. IV.4] that $X$ is bilinked via c.i.'s of type $(6,10)$ and $(6,5)$ to a degree-2 curve $Z$ satisfying $g(Z)=-2$ provided the numerical character is maximal. One may use [8, Prop. V.2] to see $h^{0}\left(\mathcal{N}_{Z}\right)=\operatorname{dim}_{(Z)} \mathrm{H}(d, g)=9$ and [23, Cor. 3.6] to compute the dimension of the bilinked family. Let us instead use that the minimal resolution of $I(Z)$ is known ( [8, Lem. V.1]). Then the mapping cone construction used twice yields a resolution:

$$
0 \rightarrow R(-10) \rightarrow R(-9)^{2} \oplus R(-8)^{2} \oplus R(-11) \rightarrow R(-8) \oplus R(-7)^{3} \oplus R(-6) \rightarrow I(X) \rightarrow 0
$$


where we have skipped a redundant term $(R(-10)$ "in the middle") since we may use the same surface of degree 6 in both linkages. This is a curve of maximal rank. To apply (11), we compute the following numbers using well known liaison formulas (e.g. [23, (2.18.1)]): $h^{1}\left(\mathcal{I}_{X}(4)\right)=h^{0}\left(\mathcal{I}_{Z}(-1)\right)=1$, $h^{1}\left(\mathcal{O}_{X}(6)\right)=4+h^{1}\left(\mathcal{O}_{Z}(1)\right)=4, h^{1}\left(\mathcal{O}_{X}(7)\right)=1+h^{1}\left(\mathcal{O}_{Z}(2)\right)=1$ and that $H^{1}\left(\mathcal{I}_{X}(v)\right)=0$ for $v \notin$ $\{4,5,6\}$. Hence $\operatorname{dim}{ }_{0} \operatorname{Hom}_{R}\left(I(X), H_{*}^{1}\left(\mathcal{O}_{X}\right)\right) \leq 7$ and then (11) implies $h^{0}\left(\mathcal{N}_{X}\right) \leq 4 d+7+1=136$. Since $\operatorname{dim} W=-d+g+56=137$ by the proven part of Theorem 3.4, we get a contradiction.

If the numerical character is not maximal we can again use [8, Lem. VI.2] (or [5]) to see that the genus of $X$ is equal to the genus of the numerical character of $X$. This implies that $X$ is smooth and ACM. By Riemann-Roch, $\chi\left(\mathcal{I}_{X}(6)\right)=4$ and $\chi\left(\mathcal{I}_{X}(7)\right)=8$. If $h^{0}\left(\mathcal{I}_{X}(6)\right)=1$, then $h^{1}\left(\mathcal{O}_{X}(6)\right)=3$, $h^{1}\left(\mathcal{O}_{X}(8)\right)=0$ and we get at least $\operatorname{dim}{ }_{0} \operatorname{Hom}_{R}\left(I(X), H_{*}^{1}\left(\mathcal{O}_{X}\right)\right) \leq 7$ (by looking at the options given by $h^{0}\left(\mathcal{I}_{X / S}(7)\right):=q, h^{1}\left(\mathcal{O}_{X}(7)\right)=4-q, 0 \leq q \leq 4$ where $S$ is defined by a degree-6 polynomial), and if $h^{0}\left(\mathcal{I}_{X}(6)\right)=2$, then a linkage via a c.i. of type $(6,6)$ yields an ACM curve of degree 4 and genus 1 , and one shows $\operatorname{dim}{ }_{0} \operatorname{Hom}_{R}\left(I(X), H_{*}^{1}\left(\mathcal{O}_{X}\right)\right)=4$. In any case we get $h^{0}\left(\mathcal{N}_{X}\right) \leq 135$ by (11), i.e. a contradiction, and the proof of this case is complete.

The case $(a, b)=(10,8)$ and $s=6$. We compute the following numbers: $(d, g)=(50,251)$, and $G(d, 7)=252$. cf. (4). Note that $g=G(d, 7)-1$. Let $X$ be a generization of $C$ satisfying $s(X)>6$. We first consider the option $s(X)=7$. If such a curve exists, we have $d(X)+r \equiv 0 \bmod 7$, i.e. $r=6=s(X)-1$. It is tempting to say that $(d, g)$ is a known Halphen's gap (cf. [5]), but before we can do that we have to compute $G(d, 8)$. We have $t^{2}-2 t+2=d$ if $t=8$, and in this part of the $B$-range (or extended $C$-range) it is known that $G(d, 8)=1+d(t-3)=251$ by (7). So strictly speaking, since $g=G(d, s(X)+1)$, it is not an Halphen's gap. We can, however, still use Ellia's results in [8] to show that $X$ does not exists. Indeed by [8, Prop. IV.3] it follows that there are no such $X$ with maximal numerical character. Since [8, Lem. VI.2] implies that the numerical character of $X$ had to be maximal, such an $X$ does not exist.

Finally we suppose $s(X) \geq 8$. Since $g=G(d, 8)$, it follows from [18, cf. (77) and [9], that $X$ is a zero section of the null correlation bundle $\mathcal{E}$; more precisely there is an exact sequence

$$
0 \longrightarrow \mathcal{O}_{\mathbb{P}^{3}} \longrightarrow \mathcal{F} \rightarrow \mathcal{I}_{X}(14) \longrightarrow 0
$$

where $\mathcal{F}=\mathcal{E}(7)$. To compute the dimension of the component $V$ of $\mathrm{H}(d, g)_{s c}$ to which $X$ belongs we will use the formula appearing in [27. Cor. 2.3] (see also Ellia's joint work with Fiorentini [10]), stating that if we have (12) and $H^{1}\left(\mathcal{I}_{X}\left(c_{1}\right)\right)=0=H^{1}\left(\mathcal{I}_{X}\left(c_{1}-4\right)\right), c_{i}:=c_{i}(\mathcal{F})$ the $i$-th Chern class, then $\mathcal{F}$ is a smooth point of its moduli space $\mathrm{M}_{\mathbb{P}^{3}}\left(c_{1}, c_{2}, c_{3}\right)$ if and only if $X$ is unobstructed and

$$
\operatorname{dim}_{(\mathcal{F})} \mathrm{M}_{\mathbb{P}^{3}}\left(c_{1}, c_{2}, c_{3}\right)+h^{0}(\mathcal{F})=\operatorname{dim}_{(X)} \mathrm{H}(d, g)+h^{0}\left(\omega_{X}\left(-c_{1}+4\right)\right) .
$$

Using this formula for $\mathcal{F}=\mathcal{E}(7)$, remarking that $H^{1}(\mathcal{E}(v))=0$ for $v \neq-1$ is well known (since a section of $\mathcal{E}(1)$ corresponds to two skew lines), we see that the assumptions for (13) to hold are fulfilled. Since $\operatorname{dim} \mathrm{M}_{\mathbb{P}^{3}}(0,1,0)=5$ and we have $h^{0}\left(\omega_{X}(-10)\right)=1$ and $h^{0}(\mathcal{F})=h^{0}(\mathcal{E}(7))=231$ by Riemann-Roch, we get $\operatorname{dim} V=\operatorname{dim}_{(X)} \mathrm{H}(50,251)=235$. Finally using that $\operatorname{dim} W=-2 d+g+$ $84+10-5=240>\operatorname{dim} V$, we get a contradiction and the proof of (i) is complete.

(iii) The component $W$ is non-reduced if we can show $\operatorname{dim} W<h^{0}\left(\mathcal{N}_{C}\right)$ for $C$ general. Since $\operatorname{dim} W=\operatorname{dim} A^{1}, H^{0}\left(\mathcal{I}_{C / S}(5)\right)=0$ and $\operatorname{dim}$ coker $\alpha_{C}=2$ it suffices by (2) to prove $h^{1}\left(\mathcal{I}_{C}(5)\right) \geq 3$. This follows from Lemma 3.3, and proof of Theorem 3.4 is complete.

Remark 3.6. Suppose that $X$ has maximal rank, or more generally that ${ }_{0} \operatorname{Ext}_{R}^{i}\left(I(X), H_{*}^{1}\left(\mathcal{I}_{X}\right)\right)=0$ for $i=0,1$ and 2. Then the formula (11) follows easily from the exact sequence [26, (2.4)] because [26. (2.1)] implies ${ }_{0} \operatorname{Ext}_{\mathfrak{m}}^{2}(I(X), I(X))=0$ and ${ }_{0} \operatorname{Ext}_{\mathfrak{m}}^{3}(I(X), I(X)) \simeq{ }_{0} \operatorname{Hom}_{R}\left(I(X), H_{*}^{1}\left(\mathcal{O}_{X}\right)\right)$ and the duality in [26, (2.2)] implies ${ }_{0} \operatorname{Ext}_{R}^{2}(I(X), I(X))^{\vee} \simeq{ }_{-4} \operatorname{Hom}_{R}\left(I(X), H_{*}^{1}\left(\mathcal{I}_{X}\right)\right)$. 
If $X$ is $A C M$, or more generally if ${ }_{0} \operatorname{Ext}_{R}^{2}(I(X), I(X))=0$ and $X$ is of maximal rank, then $X$ is unobstructed, and we may replace $h^{0}\left(\mathcal{N}_{X}\right)$ by $\operatorname{dim}_{(X)} \mathrm{H}(d, g)$ in (11) (cf. [26, Thm. 2.6], [11]).

Remark 3.7. If $s=4$ then the necessary condition $g+33 \geq 4 d$ for $W$ to be an irreducible component implies $a \geq 4$ for $d>16$ because $g=a d-2 a^{2}+1$ in Theorem 3.4. By [28, Rem. 5.3], if $D:=$ $C-4 H=x \Gamma_{1}+y \Gamma_{2}$ is effective and $h^{1}\left(S, \mathcal{O}_{S}(D)\right)>0$, then either $\Gamma_{1}$ is a fixed component of $|D|$ or $D$ is composed with a pencil. In the latter case we have $x=0$, i.e. $C=4 \Gamma_{1}+r \Gamma_{2}$ for $r \geq 6$. In a recent preprint ( [36]) Nasu shows that these curves $C$ are obstructed for every $r \geq 6$ and in the case $r=6$ where $h^{1}\left(\mathcal{I}_{C}(4)\right)=1$, he shows that $W$ is a non-reduced component of $\mathrm{H}(22,57)_{s c}$. More recently we independently finished the case $(a, b)=(7,5), s=4$ in the proof above for which $h^{1}\left(\mathcal{I}_{C}(4)\right)=2$ and $(d, g)$ attains the value $(d, g)=(22,57)$. Indeed our analysis also applies to show that $W$ is a non-reduced component in the case $r=6$. So there are at least 2 non-reduced components of $\mathrm{H}(22,57)_{s c}$ with $s=4$ (and by Theorem 4.7 one more non-reduced component for which $s=3$ ).

\section{Non-reduced components of $\mathrm{H}(d, g)_{s c}$ for $s=3$}

Motivated by the Mumford's ( [34]) example of a non-reduced component, we showed in [22] the existence of 3-maximal families that form non-reduced components of $\mathrm{H}\left(d,\left\lfloor\left(d^{2}-4\right) / 8\right\rfloor\right)_{s c}$ for every $d \geq 14$. In 22 ] we also made a conjecture about non-reduced components when $s=3$. A rough motivation for the conjecture is that the dimension of $s$-maximal families $W(s):=W$ often seems to decrease with increasing $s$, thus making the inclusion $W(s) \subset W\left(s^{\prime}\right)$ for $s^{\prime}>s$ rare without having a particular reason for such an inclusion to exist.

Conjecture 4.1. Let $W$ be a 3-maximal family of smooth connected, linearly normal space curves of degree $d>9$ and genus $g$, whose general member $C$ is contained in a smooth cubic surface. Then $W$ is a non-reduced irreducible component of $\mathrm{H}(d, g)_{s c}$ if and only if

$$
d \geq 14, \quad 3 d-18 \leq g \leq\left(d^{2}-4\right) / 8 \text { and } H^{1}\left(\mathcal{I}_{C}(3)\right) \neq 0 .
$$

Note that a 3-maximal family $W$ is closed and irreducible by our definition, and that $\operatorname{dim} W=$ $d+g+18$ holds for $d>9$. The above conjecture, originating in [22], is here presented by modifications proposed by Ellia [7] (see also [6] by Dolcetti, Pareschi), because they found counterexamples which heavily depended on the fact the general curves $C$ were not linearly normal (i.e. $\left.H^{1}\left(\mathcal{I}_{C}(1)\right) \neq 0\right)$.

The conjecture is known to be true in many cases. Indeed Mumford's example of a non-reduced component is in the range of Conjecture 4.1 (minimal with respect to both degree and genus). Also the main result by the author in [21] shows the conjecture provided

$$
g>7+(d-2)^{2} / 8, \quad d \geq 18 .
$$

Ellia shows in 7 that Conjecture 4.1 holds in the larger range $g>G(d, 5), d \geq 21$ by first proving

Proposition 4.2. (Ellia) Let $d \geq 21$ and $g \geq 3 d-18$, let $W$ be a 3-maximal family of smooth connected space curves whose general curve $C$ sits on a smooth surface and suppose that $H^{1}\left(\mathcal{I}_{C}(1)\right)=$ 0 . If $X$ is a generization of $C$ in $\mathrm{H}(d, g)_{\text {sc }}$ satisfying $H^{0}\left(\mathcal{I}_{X}(3)\right)=0$, then $H^{0}\left(\mathcal{I}_{X}(4)\right)=0$.

See [7, Prop. VI.2] for a proof. More recently Nasu proves (and reproves) a part of the conjecture by showing that the cup-product (i.e the primary obstruction) is nonzero if $h^{1}\left(\mathcal{I}_{C}(3)\right)=1$ ( [35]).

When we try to show that generizations $X$ of a curve $C$ with $s(C)=s-1$ do not exist, the hard part is usually the case $s(X)=s$. The non-existence of such $X$ are taken care of by Proposition 4.2 for $s=4$. Therefore combining Ellia's result with semi-continuity arguments when $s(X)>s$, we 
can extend the range where Conjecture 4.1 holds in a good number of cases. This is what we do in the Theorems 4.4 and 4.5. Recalling that we can associate a curve $C$ on $S$ and its corresponding invertible sheaf $\mathcal{O}_{S}(C)$ with a 7 -tuple of integers $\left(\delta, m_{1}, . ., m_{6}\right)$ satisfying (15) below (by blowing up $\mathbb{P}^{2}$ in six general points in the usual way, cf. [17]), we first remark (cf. [21, Lem. 16 and Cor. 17])

Lemma 4.3. With notations as above it holds

(i) $d=3 \delta-\sum_{i=1}^{6} m_{i}, \quad g=\left(\begin{array}{c}\delta-1 \\ 2\end{array}\right)-\sum_{i=1}^{6}\left(\begin{array}{c}m_{i} \\ 2\end{array}\right)$.

(ii) If $g>\left(d^{2}-4\right) / 8$, then $H^{1}\left(\mathcal{I}_{C}(3)\right)=0$, whence $C$ is unobstructed.

(iii) If $d \geq 14$ and $g \geq 3 d-18$, then $H^{1}\left(\mathcal{I}_{C}(3)\right) \neq 0$ and $H^{1}\left(\mathcal{I}_{C}(1)\right)=0 \Leftrightarrow 1 \leq m_{6} \leq 2$.

By Lemma 4.3 (ii) the conditions of Conjecture 4.1 are necessary for $W$ to be a non-reduced component, and for the converse, and we may suppose $m_{6}=1$ or 2 by (iii). If $m_{6}=1$ we recall

Theorem 4.4. Let $W$ be a 3-maximal family of smooth connected space curves, whose general member sits on a smooth cubic surface and corresponds to the 7 -tuple $\left(\delta, m_{1}, . ., m_{6}\right)$ satisfying

$$
\delta \geq m_{1} \geq . . \geq m_{6} \text { and } \delta \geq m_{1}+m_{2}+m_{3} .
$$

Then $W$ is a non-reduced irreducible component of $\mathrm{H}(d, g)_{\text {sc }}$ provided

a) $m_{6}=1, m_{5} \geq 6, d \geq 35$ and $\left(\delta, m_{1}, . ., m_{6}\right) \neq(\lambda+18, \lambda+6,6, . ., 6,1)$ for any $\lambda \geq 2$, or

b) $m_{6}=1, m_{5}=5, m_{4} \geq 7, d \geq 35$ and $\left(\delta, m_{1}, . ., m_{6}\right) \neq(\lambda+21, \lambda+7,7, . ., 7,5,1)$ for $\lambda \geq 2$.

For a proof, see the appendix to [28, Thm. A.3] by the first author. For $m_{6}=2, m_{5} \geq 3$ see [35]. It is also possible to use the idea of [21, Sect.4] to determine bounds for $\operatorname{dim} V$ where $V \supset W$ and $\operatorname{dim} V>\operatorname{dim} W$. This is done in [28, Sect.4], leading to [28, Prop. A.7] which we will generalize and completely prove. Note that by Proposition 4.2 we can skip considering components with $s(V)=4$.

Theorem 4.5. Let $W$ be a 3-maximal family of smooth connected space curves, whose general member is linearly normal and sits on a smooth cubic surface. If

$$
g>\max \left\{\frac{d^{2}}{10}-\frac{d}{2}+18, G(d, t)\right\}, d \geq t^{2}-2 t+2,
$$

for some $t$ satisfying $6 \leq t \leq 8$, then $W$ is an irreducible component of $\mathrm{H}(d, g)_{s c}$. Moreover, $W$ is non-reduced if and only if $H^{1}\left(\mathcal{I}_{C}(3)\right) \neq 0$. In particular Conjecture 4.1 holds in the range (16), e.g. if

$$
g>\frac{d^{2}}{10}-\frac{d}{2}+18, d \geq 54
$$

We have $G(d, 6) \geq \frac{d^{2}}{10}-\frac{d}{2}+18$ if and only if $d \leq 74$. This is one of the reasons for proving (16) not only for $t=6$, but also $t=7$ and 8 since it enlarges the range where the conjecture holds. Note also that Ellia proves the conjecture for $g>G(d, 5)=\frac{d^{2}}{10}+\frac{d}{2}+1-\frac{2 r(5-r)}{5}$, so the improvement in Theorem 4.5 is only of order $d$. The improvement in Theorem 4.4 is more substantial.

In the proof of Theorem 4.5 we will need (see [28, Prop. 4.4] for a proof):

Proposition 4.6. Let $V$ be an irreducible component of $\mathrm{H}(d, g)_{s c}$ whose general curve $C$ sits on some integral surface $F$ of degree $s \geq 4$. If $d>s^{2}$, then

$$
\operatorname{dim} V \leq\left(\begin{array}{c}
s+3 \\
3
\end{array}\right)-1+\max \left\{\frac{d^{2}}{s}-g, \frac{d^{2}}{2 s},(4-s) d+g-1+h^{0}\left(\mathcal{O}_{C}(s-4)\right)\right\} .
$$


Proof (of Theorem 4.5). To see that $W$ is an irreducible component, we suppose there exists a component $V$ of $\mathrm{H}(d, g)_{s c}$ satisfying $W \subset V$ and $\operatorname{dim} W<\operatorname{dim} V$. Then $s(V) \geq 4$ by the definition of a 3-maximal family, whence $s(V) \geq 5$ by Proposition 4.2 . Firstly suppose $g>G(d, 6)$ and $d \geq 26$. It follows that the general curve $X$ of $V$ satisfies $s:=s(X)=5$. To get a contradiction we will use Proposition 4.6 for $s=5$ and the fact $\operatorname{dim} W=d+g+18$. Indeed since $X$ is a generization of a smooth connected linearly normal curve, it follows that a surface containing $X$ of the least possible degree is integral and moreover that $X$ is smooth, connected and linearly normal. We have

$$
d+g+18<55+\max \left\{\left\lfloor\frac{d^{2}}{5}\right\rfloor-g,\left\lfloor\frac{d^{2}}{10}\right\rfloor,-d+g-1+4+h^{1}\left(\mathcal{I}_{X}(1)\right)\right\} .
$$

Suppose the maximum to the right is attained by $55-d+g-1+4$. Then $d+g+18<55-d+g+3$ which is absurd since we have assumed $d>25$. Similarly if the maximum is attained by $55+\left\lfloor d^{2} / 5\right\rfloor-g$ we get $d+g+18<55+\left\lfloor d^{2} / 5\right\rfloor-g$ or equivalently $2 g \leq 36+\left\lfloor d^{2} / 5\right\rfloor-d$ which contradicts the assumption $g>\frac{d^{2}}{10}-\frac{d}{2}+18$. Also $d+g+18<55+\left\lfloor d^{2} / 10\right\rfloor$ leads to a contradiction because $36+\frac{d^{2}}{10}-d \leq \frac{d^{2}}{10}-\frac{d}{2}+18$ for $d \geq 36$ and $36+\frac{d^{2}}{10}-d \leq G(d, 6)$ for $26 \leq d<36$. For the latter inequality, we remark that we, for $26 \leq d<31$, have to compute $G(d, 6)$ in the $B$-range (or "extended $C$-range") using (6) and (7). Thus we have proved that $W$ is an irreducible component of $\mathrm{H}(d, g)_{s c}$.

Secondly we suppose $g>G(d, 7)$ and $d \geq 37$. It follows from the previous paragraph that the assumptions $s(X)=5$ and $g>\frac{d^{2}}{10}-\frac{d}{2}+18$ yield a contradiction. Hence we may assume $s(X)=6$. Now if we use Proposition 4.6 for $s:=s(X)=6$, which requires $d>36$, we get

$$
d+g+19 \leq 83+\max \left\{\left\lfloor\frac{d^{2}}{6}\right\rfloor-g,\left\lfloor\frac{d^{2}}{12}\right\rfloor,-2 d+g-1+h^{0}\left(\mathcal{O}_{X}(2)\right)\right\} .
$$

To estimate $h^{0}\left(\mathcal{O}_{X}(2)\right)$ we use Clifford's theorem to see $h^{0}\left(\mathcal{O}_{X}(2)\right)-1 \leq \max \{2 d-g, d\}$. Since it is easy to see

$$
\max \left\{32+\frac{1}{2}\left\lfloor\frac{d^{2}}{6}\right\rfloor-\frac{d}{2}, 64+\left\lfloor\frac{d^{2}}{12}\right\rfloor-d\right\} \leq \frac{d^{2}}{10}-\frac{d}{2}+18
$$

for $d \geq 40$, we have a contradiction except for $37 \leq d \leq 39$. For these exceptions in the extended $C$-range we compute $G(d, 7)$ by using (6) and (7) and we get a contradiction since $g>G(d, 7)$ for $37 \leq d \leq 39$. Again we can conclude that $W$ is an irreducible component of $\mathrm{H}(d, g)_{s c}$.

Thirdly we suppose $g>G(d, 8)$ and $d \geq 50$. It follows from the previous paragraph that the assumptions $s(X)=6$ (or 5 ) and $g>\frac{d^{2}}{10}-\frac{d}{2}+18$ yield a contradiction. We may therefore assume $s(X)=7$. Now using Proposition 4.6 for $s:=s(X)=7$, which requires $d>49$, we get

$$
d+g+19 \leq 119+\max \left\{\left\lfloor\frac{d^{2}}{7}\right\rfloor-g,\left\lfloor\frac{d^{2}}{14}\right\rfloor,-3 d+g-1+h^{0}\left(\mathcal{O}_{X}(3)\right)\right\} .
$$

We use Clifford's theorem to get $h^{0}\left(\mathcal{O}_{X}(3)\right)-1 \leq \max \{3 d-g, 3 d / 2\}$. Since it is rather easy to see

$$
\max \left\{50+\frac{1}{2}\left\lfloor\frac{d^{2}}{7}\right\rfloor-\frac{d}{2}, 100+\left\lfloor\frac{d^{2}}{14}\right\rfloor-d\right\} \leq \frac{d^{2}}{10}-\frac{d}{2}+18
$$

for $d \geq 46$, we have a contradiction, i.e. $W$ is an irreducible component of $\mathrm{H}(d, g)_{s c}$.

Since $G(d, 8) \leq \frac{d^{2}}{10}-\frac{d}{2}+18$ for $d \geq 58$ and $d \in\{54,55,56\}$, then $W$ is an irreducible component (again we need to work in the extended $C$-range).

Finally for $d=57, g=G(d, 8)=315$ we check $\operatorname{dim} W \geq \operatorname{dim} V$ directly. Indeed the general curve $X$ of $V$ is by (5) directly linked via a c.i. of type $(8,8)$ to a plane curve $X^{\prime}$ of degree $r=7$ since $d+r \equiv 0 \bmod 8$. The mapping cone construction yields a minimal resolution $0 \rightarrow R(-15) \oplus R(-9) \rightarrow$ $R(-8)^{3} \rightarrow I(X) \rightarrow 0$, and since well known linkage formulas imply $h^{1}\left(\mathcal{O}_{X}(8)\right)=h^{0}\left(\mathcal{I}_{X^{\prime}}(4)\right)=20$ and $h^{1}\left(\mathcal{O}_{X}(9)\right)=10$, we get $\operatorname{dim} V=4 d+3 h^{1}\left(\mathcal{O}_{X}(8)\right)-h^{1}\left(\mathcal{O}_{X}(9)\right)=285$ by (11) while $\operatorname{dim} W=$ $d+g+18=390$, a contradiction. Thus (17) imply that $W$ is an irreducible component of $\mathrm{H}(d, g)_{s c}$. 
Then using $\operatorname{dim} W+h^{1}\left(\mathcal{I}_{C}(3)\right)=h^{0}\left(\mathcal{N}_{C}\right)$, cf. (2) , we get the final statement and we are done.

What still lacks in this picture are existence results of components in the range of the (proven part of the) conjecture. It is, however, not so difficult to prove existence. One may, as Dolcetti and Pareschi do in [6], prove existence using Rathmann's work [37]. By [37] one knows that there exists a smooth connected curve contained in a smooth del Pezzo surface in $\mathbb{P}^{4}$, of degree $d>4$ and genus $g$ provided

$$
(d+12) \sqrt{d+9}-11 d / 2-35 \leq g \leq 1+\left(d^{2}-4 d\right) / 8 .
$$

Indeed Rathmann shows the existence of a 6 -tuple $\left(\delta, m_{1}, . ., m_{5}\right)$ satisfying $\mathcal{O}_{S}(C)^{2}>0$ and $\delta \geq$ $m_{1} \geq . . \geq m_{5} \geq 0$ and $\delta \geq m_{1}+m_{2}+m_{3}$ for every $(d, g)$ in the range (18). Letting $m_{6}=0$ this leads to a 7-tuple $\left(\delta, m_{1}, . ., m_{5}, m_{6}\right)$ satisfying (15) for every $(d, g)$ given as in Lemma 4.3 (i) in the range (18), whence we get the existence of a smooth connected curve $C$ sitting on our smooth cubic surface $S \subset \mathbb{P}^{3}$. If we now add a 7 -tuple corresponding to $n$ hyperplanes, $(3 n, n, . . n)$, to $\left(\delta, m_{1}, . ., m_{5}, 0\right)$, the corresponding linear system will contain smooth connected curves $X \in|C+n H|$ satisfying $m_{6}=n$ and $H^{1}\left(I_{X}(v)\right)=0$ for every non-negative integer $v \leq n$. Using the adjunction formula for the genus we easily see that the degree $d^{\prime}$ and genus $g^{\prime}$ of $X$ satisfy

$$
d=d^{\prime}-3 n \text { and } g=-n d^{\prime}+g^{\prime}+3\left(n^{2}+n\right) / 2 .
$$

Inserting these formulas into (18) we get for every $d^{\prime}>3 n+4, g^{\prime}$ in the range

$$
\left(d^{\prime}+12-3 n\right) \sqrt{d^{\prime}+9-3 n}+d^{\prime}\left(n-\frac{11}{2}\right)-35+\frac{3\left(10 n-n^{2}\right)}{2} \leq g^{\prime} \leq 1+\frac{d^{\prime 2}+(2 n-4) d^{\prime}-3 n^{2}}{8}
$$

the existence of such a curve $X$. Using this for $n=1$ and $n=2$ we get the following result.

Theorem 4.7. For every $d \geq 14$ and $g,(d, g) \neq(14,22)$ in the range

$$
(d+9) \sqrt{d+6}-\frac{9 d}{2}-\frac{43}{2} \leq g \leq \frac{d^{2}-4}{8}
$$

there exists a smooth connected curve $C$ of degree $d$ and genus $g$, contained in a smooth cubic surface in $\mathbb{P}^{3}$, whose corresponding 7 -tuple $\left(\delta, m_{1}, . ., m_{6}\right)$ has $m_{6}$ equal to 1 or 2 and such that $\left(\delta, m_{1}, . ., m_{6}\right) \neq\left(\lambda+3 m_{6}, \lambda+m_{6}, m_{6}, . ., m_{6}\right)$ for every $\lambda \geq 2$. If also $g \geq 3 d-18$ holds, then $C$ satisfies

$$
H^{1}\left(\mathcal{I}_{C}(3)\right) \neq 0 \text { and } H^{1}\left(\mathcal{I}_{C}(1)\right)=0 .
$$

In particular for every $d, g \leq \frac{d^{2}-4}{8}$ satisfying either (14), (16), (17) or $g>G(d, 5), d \geq 21$ there exists a non-reduced component $W$ of $\mathrm{H}(d, g)_{s c}$ whose general curve sits on a smooth cubic hypersurface.

Proof. Let $G 1:=\frac{d^{2}-2 d+5}{8}, G 2:=\frac{d^{2}-4}{8}$, and let

$$
g 1:=(d+9) \sqrt{d+6}-\frac{9 d}{2}-\frac{43}{2}, g 2:=(d+6) \sqrt{d+3}-\frac{7 d}{2}-11 .
$$

Using (19) with $n=1$ (resp. $n=2$ ) we get the existence of a smooth connected curve $C$ for every $(d, g)$ in the range $g 1 \leq g \leq G 1$ (resp. $g 2 \leq g \leq G 2)$ with a 7 -tuple with the desired properties. One verifies that $g 1 \leq g 2 \leq G 2$ for $d \geq 14$ and that $g 2<G 1<G 2$ for $d \geq 17$. Since the only integer $k$ satisfying $G 1<k<g 2$ for $14 \leq d \leq 16$ is $k=22$ in which case $d=14$, we get the first conclusion of the theorem. Moreover invoking Lemma 4.3 (iii) we get the next conclusion. Finally looking at the statements accompanying (14), (16), (17) or $g>G(d, 5), d \geq 21$, we see that the conjecture holds in these ranges, and we get the final conclusion of the theorem. 
Remark 4.8. (i) For $d \geq 17$ we have $g 1 \leq g 2 \leq G 1 \leq G 2$, with notations as in the proof. If $d, g$ satisfy $g 2 \leq g \leq G 1$ it follows from the proof that there exists two 7-tuples, one with $m_{6}=1$ and one with $m_{6}=2$ with $d, g$ satisfying (20). If we in addition are in a range of the proven part of the conjecture, we have two different 3-maximal families that form non-reduced components of $\mathrm{H}(d, g)_{s c}$. (ii) If we use (19) for $n=3$ we get the existence of a smooth connected curve $C$ for every $d \geq 14$ and $g$ in the range

$$
(d+3) \sqrt{d}-\frac{5 d}{2}-\frac{7}{2} \leq g \leq \frac{d^{2}+2 d-19}{8},
$$

with $m_{6}=3$ and $\left(\delta, m_{1}, . ., m_{6}\right) \neq(\lambda+9, \lambda+3,3, . .3)$ for any $\lambda \geq 2$. Such $C$ belongs to a generically smooth, irreducible component of $\mathrm{H}(d, g)_{\text {sc }}$ because $H^{1}\left(\mathcal{I}_{C}(3)\right)=0$. This improves upon the lower bound of [25, Prop. 3.1] for $d \gg 0$. Indeed [25, Prop. 3.1], which states that for $d>9$ and $(d, g)$ satisfying

$$
3 d-17+\frac{(d-9)(d-18)}{18} \leq g \leq 1+\frac{d(d-3)}{6}, \quad \text { and }
$$

$(d, g) \notin\{(30,91),(33,103),(34,109)\}$ there exists an unobstructed curve $C$ of $\mathrm{H}(d, g)_{\text {sc }}$ contained in a smooth cubic surface, gives a better lower bound for $9<d \leq 161$. The proof of [25, Prop. 3.1] which makes use of Gruson and Peskine's existence result [17, Prop. 2.10], produces a curve C with 7-tuple satisfying $m_{6} \geq 3$ and such that $H^{1}\left(\mathcal{I}_{C}(v)\right)=0$ for $v \leq 3$ for every $(d, g)$ in the mentioned range, thus $C$ belongs a 3-maximal family $W$ which is a generically smooth component of $\mathrm{H}(d, g)_{s c}$.

\section{References}

[1] C. Ciliberto, A.F. Lopez. On the existence of components of the Noether-Lefschetz Locus with given codimension. Manuscripta Math. 73 no. 4 (1991), 341-358.

[2] D. J. Curtin Obstructions to deforming a space curve. Trans Amer. Math. Soc, 267 (1981), 83-94.

[3] A. Dan. Non-reduced components of the Noether-Lefschetz locus. Preprint arXiv.org/abs/1407.8491v2.

[4] A. Dan. Noether-Lefschetz locus and generalisation of an example due to Mumford. Preprint arXiv.org/abs/1409.6012.

[5] A. Dolcetti. Halphen's gaps for space curves of submaximum genus. Bulletin de la S. M. F. 116 no. 2 (1988), 157-170.

[6] A. Dolcetti, G. Pareschi. On Linearly Normal Space Curves. Math. Z. 198 (1988), 73-82.

[7] Ph. Ellia: D'autres composantes non réduites de Hilb $\mathbb{P}^{3}$. Math. Ann. 277 (1987), 433-446.

[8] Ph. Ellia: Sur les lacunes d'Halpen, in "Algebraic Curves and Projective Geometry, Proceedings (Trento, 1988)," Lectures Notes in Math., Vol. 1389 Springer-Verlag, 1989.

[9] Ph. Ellia Sur le genre maximal des courbes de degre d non sur une surface de degre $s-1$. J. reine angew. Math. 413 (1991), 78-87.

[10] Ph. Ellia and M. Fiorentini, Quelques remarques sur les courbes arithmetiquement Buchsbaum de l'espace projectif. Ann. Univ. Ferrara XXXII (1987), 89-111.

[11] G. Ellingsrud. Sur le schéma de Hilbert des variétés de codimension 2 dans $\mathbb{P}^{e}$ a cône de Cohen-Macaulay. Ann. Scient. Éc. Norm. Sup. 8 (1975), 423-432.

[12] G. Ellingsrud and C. Peskine. Anneau de Gorenstein associé à un fibré inversible sur une surface de l'espace et lieu de Noether-Lefschetz. Proceedings of the Indo-French Conference on Geometry (Bombay, 1989), 29-42, Hindustan Book Agency, Delhi, 1993.

[13] G. Fløystad. Determining obstructions for space curves, with application to non-reduced components of the Hilbert scheme. J. reine angew. Math. 439 (1993), 11-44.

[14] M. Green. A new proof of the explicit Noether-Lefschetz theorem. J. Diff. Geom. 27 (1988), 155-159.

[15] A. Grothendieck. Les schémas des Picard: théorème d'existence. Séminaire Bourbaki, exp. 232 (1961/62).

[16] L. Gruson, Chr. Peskine. Genre des courbes de l'espace projectif, in: Proc. Tromsø 1977, Lectures Notes in Math., Vol. 687 (1978), Springer-Verlag, New York, 1986.

[17] L. Gruson, Chr. Peskine. Genre des courbes de l'espace projectif (II). Ann. Sci. Éc. Norm. Sup. (4) 15 (1982), 401-418. 
[18] L. Gruson, Chr. Peskine. Postulation des courbes gauches, in: Proc. Ravello 1982, Lectures Notes in Math., Vol. 997 (1983), Springer-Verlag, New York, 1986. pp 218-227.

[19] R. Hartshorne. Stable reflexive sheaves III. Math. Ann. 279 (1988), 517-534.

[20] R. Hartshorne. Deformation theory. Graduate Texts in Math. Springer-Verlag, New York, 2010.

[21] J.O. Kleppe. Non-reduced components of the Hilbert scheme of smooth space curves, in Proceedings Rocca di Papa 1985 Lectures Notes in Math., Vol. 1266 Springer-Verlag, 1987.

[22] J.O. Kleppe. The Hilbert-flag scheme, its properties and its connection with the Hilbert scheme. Applications to curves in 3-space. Preprint (part of thesis), March 1981, Univ. of Oslo. https://www.cs.hioa.no/ jank/papers.htm (new address, which replaces http://www.iu.hio.no/ jank/papers.htm).

[23] J.O. Kleppe. Liaison of families of subschemes in $\mathbb{P}^{n}$, in "Algebraic Curves and Projective Geometry, Proceedings (Trento, 1988)," Lectures Notes in Math., Vol. 1389 Springer-Verlag, 1989.

[24] J.O. Kleppe. Halphen Gaps and Good Space Curves. Boll. U.M.I (8) 1-B (1998), 429-450.

[25] J.O. Kleppe. On the existence of Nice Components in the Hilbert Scheme $\mathrm{H}(d, g)$ of Smooth Connected Space Curves. Boll. U.M.I (7) 8-B (1994), 305-326.

[26] J.O. Kleppe. The Hilbert Scheme of Space Curves of small diameter. Annales de l'institut Fourier 56 no. 5 (2006), 1297-1335.

[27] J.O. Kleppe. Moduli spaces of reflexive sheaves of rank 2. Canad. J. Math. 62 (2010), 1131-1154.

[28] J.O. Kleppe and John C. Ottem. Components of the Hilbert scheme of space curves on low-degree smooth surfaces. International Journal of Mathematics, Vol.26, no.2 (2015).

[29] A. Laudal. Formal Moduli of Algebraic Structures. Lectures Notes in Math., Vol. 754, Springer-Verlag, New York, 1979.

[30] A. Lopez. Noether-Lefschetz theory and the Picard group of Surfaces. Mem. Amer. Math. Soc. 89 (1991).

[31] M. Martin-Deschamps, D. Perrin. Sur la classification des courbes gauches. Asterisque, 184-185 (1990).

[32] M. Martin-Deschamps, D. Perrin. Le schéma de Hilbert des Courbes Gauches localement CohenMacaulay n'est (presque) jamais reduit. Ann. Scient. Éc. Norm. Sup. 4 t. 29 (1996), 757-785.

[33] S. Mukai and H. Nasu. Obstructions to deforming curves on a 3-fold, I: A generalization of Mumford's example and an application to Hom schemes. Journal of Algebraic Geometry 18 (2009), 691-709.

[34] D. Mumford. Further pathologies in algebraic geometry. Amer. J. Math. 84 (1962), 642-648.

[35] H. Nasu. Obstructions to deforming space curves and non-reduced components of the Hilbert scheme. Publ. Res. Inst. Math. Sci. 42 (2006), no 1, 117-141.

[36] H. Nasu. Obstructions to deforming curves on a 3-fold, III: Deformations of curves lying on a K3 surface. Preprint. arXiv.org/abs/1601.07301.

[37] J. Rathmann The Genus of Curves in $\mathbb{P}^{4}$ and $\mathbb{P}^{5}$. Math. Z. 202 (1989), 525-543.

[38] C. Voisin. Composantes de petite codimension du lieu de Noether-Lefschetz. Comment. Math. Helv. 64 no.4 (1989), 515-526.

OSLO AND AKERSHUS UNIVERSITY COLLEGE OF APPLIED SCIENCES, FACULTY OF TECHNOLOGY, ART AND DESIGN, PO BOX 4 ST. OLAVS PLASS, NO-0130 OSLO, NORWAY.

E-mail address: JanOddvar.Kleppe@hioa.no 